\title{
Swarms Robots and their applications
}

\author{
Yazdani Hasan \\ Department of Computer Application Sri Satya Sai University of Technology \& Medical Sciences, Bhopal, \\ India.
}

\begin{abstract}
Swarm robotics is a new approach to the coordination of multirobot systems which consist of large numbers of mostly simple physical robots In this paper, we will discuss this emerging filed, Swarm Robots. This filed has many applications. We will also discuss these applications in detail.
\end{abstract}

Keywords: Robot, Intelligence, Swarm, Applications.

\section{Introduction}

Swarm robotics is a new approach to the coordination of multirobot systems which consist of large numbers of mostly simple physical robots. The desired collective behaviour emerges from the interactions between the robots and interactions of robots with the environment. This approach emerged on the field of artificial swarm intelligence, as well as the biological studies of insects, ants and other fields in nature, where swarm behaviour occurs. Individuals within the group interact by exchanging locally available information such that the problem (global objective) is solved more efficient than it would be done by a single individual. Problem-solving behaviour that emerges from such interactions is called swarm intelligence [1-5].Swarm robotics is the application of swarm intelligence techniques to the analysis of activities in which the agents are physical robotic devices that can effect changes in their environments based on intelligent decision-making from various input. The goal of this approach is to study the design of robots such that a desired collective behaviour emerges from the inter-robot interactions and the interactions of the robots with the environment, inspired but not limited by the emergent behaviour observed in social insects.The paper is organized in the following manner. The Section 2 has discussion about the swarms robots. Section 3 has application of swarm robots. Section 4 has conclusion and future work.

\section{Swarm Robots}

In this section we will discuss some of the properties of swarm robots.There are many examples of collectively complex behaviour for example social insects such as: ant' colonies, termites, bees, wasps ...etc., So, a swarm intelligence system consists typically of a population of relatively simple agents interacting only locally with themselves and with their environment, without having a global knowledge about their own state and of the state of the world [4-9].

The swarm robots have following properties.

1- Autonomy -individuals that create the swarm-robotic system are autonomous robots. They are independent and can interact with each other and the environment..

2- Large number - They are in large number so they can cooperate with each other.

3- Scalability and robustness - A new unit can be easily added to the system so the system is easily scalable. More number of units improve the performance of the system. The system is quite robust to the loosing some units as there still exists some units left to perform. Though the system will not perform up to its maximum capabilities.

4- Decentralized coordination - The robots communicate with each other and with environment to take the final decision.

5- Flexibility- It requires the swarm robotic system to have the ability to generate modularized solutions to different tasks

\section{Appications}

Swarm robots have many applications [1-9].Disaster rescue missions is one of the most important applications of swarms robots. Swarms of robots of could be sent to places rescue workers can't reach this would save lives. Swarm robots have many applications in mining tasks Swarms robots can be used in military to form an autonomous army. Swarm robots are also useful for autonomous surveillance and environment monitoring to investigate environmental parameters, search for survivors, and locate sources of hazards such as chemical or gas spills, toxic pollution, pipe leaks, radioactivity. Swarm robots can perform tasks in which the main goal is to cover a wide region. The robots can disperse and perform monitoring tasks, for example, in forests. It can be useful for detecting hazardous events, like a leakage of a chemical substance. In Military 
applications, it is very much useful in detecting bomb which is most dangerous task for the humans. Robotics is expected to play a major role in the agricultural/farming domain. Swarm robotics, in particular, is considered extremely relevant for precision farming and large-scale agricultural applications.. Specifically, a decentralised monitoring/mapping scenario, and implement a use case for the detection and mapping of weeds in a field by a group of small unmanned aerial vehicles (UAVs).

Toxic waste clean-up, search and rescue (SAR) and collection of terrain samples are some of the important applications of Swarms robots. Swarms robots can be for Exploration and mapping it would save money and time. Presently, most of the efforts are being made in surveillance, reconnaissance and hazard detection.In medical fields, a use of nano-robots moving through human veins and arteries (e.g. to fight certain types of cancer). There are many industries which use dangerous things like burning furnace, chemicals, making nuclear weapons etc. Here usage of swarms can reduce danger in such types of industries. The possible real applications of swarm robotics will take special importance when robots get to be mass produced and the costs of building swarms of robots decrease. The development of technologies such as MEMS (Micro-ElectroMechanical Systems) will allow to create small and cheap robots.In this way swarms of robots can be really useful for dangerous tasks.For example, for mining detection and cleaning. The number of possible applications is really promising, but still the technology must firstly be developed both in the algorithmic and modelling part, and also in the miniaturisation technologies.

\section{Conclusion}

In this paper, we discussed about the basics of Swarms Robots. We also discussed about the applications of Swarms Robots. This is an emerging field. In future, it will have many new applications.

\section{References}

[1]. Camazine, S., Deneubourg, J.L., Franks, N., Sneyd, J., Theraulaz, G., Bonabeau, E.: Self-Organisation in Biological Systems. Princeton University Press, NJ, USA (2001)

[2]. Beni, G., Wang, J.: Swarm intelligence. In: Proc. of the Seventh Annual Meeting of the, Tokyo, Japan (1989) $425-428$.

[3]. Beni, G.: From swarm intelligence to swarm robotics. In $S_{s}$ ahin, E., Spears, W., eds.: Swarm Robotics: State-of-the-art Survey. Lecture Notes in Computer Science 3342, Springer-Verlag (2005) 1-9.

[4]. Bonabeau, E., Dorigo, M., Theraulaz, G.: Swarm Intelligence: From Natural to Artificial Systems. Oxford University Press, New York, NY, USA (1999).

[5]. Dudek, G., Jenkin, M., Milios, E., Wilkes, D.: A taxonomy for multi-agent robotics. Autonomous Robots 3 (1996) $375-397$.

[6]. Cao, Y., Fukunaga, A., Kahng, A.: Cooperative mobile robotics: Antecedents and directions. Autonomous Robots 4 (1997) 1-23.

[7]. Kube, C., Zhang, R.: Collective robotics: From social insects to robots. Adaptive Behavior 2 (1994) $189-218$.

[8]. M. Schwager, J. McLurkin, J. J. E. Slotine, and D. Rus, "From theory to practice: distributed coverage control experiments with groups of robots," in Proceedings of International Symposium on Experimental Robotics, Athens, Greece, July 2008.

[9]. I. Navarro and F. Matía, "A survey of collective movement of mobile robots," Tech. Rep., Universidad Polit ecnica de Madrid, 2010 . 